\title{
La fecundidad de las mujeres latinoamericanas y caribeñas en España: ¿adaptación, mantenimiento 0 interrupción?*
}

\author{
Rafael Grande y Alberto del Rey
}

Universidad de Málaga, España/Universidad de Salamanca, España

\section{Resumen}

El objetivo de este trabajo es analizar la fecundidad de las mujeres inmigrantes procedentes de América Latina y el Caribe en España, poniéndola en relación con la fecundidad en el origen y en el destino. Así se aborda el debate sobre la integración de las mujeres latinas observando si optan por mantener su comportamiento reproductivo y, por lo tanto, el nivel de fecundidad existente en su país de origen o por el contrario, si se adaptan al de la sociedad de destino. Adoptando una perspectiva de análisis longitudinal y usando modelos multivariables de supervivencia con datos procedentes de la Encuesta Nacional de Inmigrantes 2007, los resultados muestran la convivencia de procesos de adaptación, mantenimiento e interrupción en la fecundidad inmigrante. Destaca la importancia de las condiciones familiares a la llegada en el comportamiento reproductivo.

Palabras clave: Inmigración, fecundidad, integración, latinoamericanos, España.

\section{Abstract}

The fertility of Latin American and Caribbean women in Spain: adaptation, maintenance or interruption?

The aim of this paper is to analyze the fertility behavior of immigrant women from Latin America and the Caribbean in Spain, looking for the existence of relationship between fertility in home and destination countries. Particularly, we discuss the integration of Latin-American women exploring whether they choose to keep their reproductive behavior and, therefore, the existing level of fertility in their country of origin or whether, conversely, they adapt to the fertility patterns of the country of destination. Adopting a longitudinal perspective and applying multivariate survival models to the data of the 2007 National Survey of Immigration, we show the coexistence of adaptation, maintenance and interruption patterns in the immigrant fertility. Our results highlights the relevance of family conditions on arrival in reproductive behavior.

Key words: Immigration, fertility, integration, Latin-Americans, Spain.

* Trabajo financiado por los proyectos de investigación CSO2013-41828-R titulado "Trayectorias laborales y reproductivas de la población inmigrante en tiempo de crisis" y CSO201457410-JIN titulado"Asimilación laboral e integración social en flujos migratorios sur-sur y surnorte. El caso de América Latina y el Caribe" del Ministerio de Economía y Competitividad, España. 


\section{INTRODUCCIÓN}

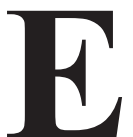

1 estudio de la fecundidad inmigrante es, desde hace décadas, un objeto de estudio muy relevante en las ciencias sociales en general y en la demografía en particular. Por un lado, porque es un factor determinante de las dinámicas demográficas, tanto en las sociedades de origen como especialmente en las sociedades de destino por el hecho de que en general estas sociedades receptoras suelen presentar bajos niveles de fecundidad. Por otro lado, la fecundidad de la población inmigrante se ha constituido en un importante indicador del grado de integración de los diferentes colectivos de inmigrantes en las sociedades de acogida.

En esta segunda perspectiva es donde ubicamos nuestro trabajo. El objetivo es analizar si la fecundidad de las mujeres latinoamericanas que han emigrado a España se ajusta al patrón de las mujeres españolas, tanto en su intensidad como en su calendario, o por el contrario, revela el comportamiento reproductivo de sus sociedades de origen.

Nos centramos exclusivamente en la población latinoamericana y caribeña por haber sido el principal colectivo durante el boom inmigratorio de principios del siglo XXI en España (Muñoz de Bustillo y Antón, 2010). Un colectivo que, al mismo tiempo que presenta una cierta homogeneidad cultural en relación a la sociedad de acogida, presenta una alta heterogeneidad respecto a los factores determinantes de su comportamiento reproductivo, como son las características personales y familiares, o la fase de transición demográfica de la sociedad de origen (Roig-Vila y Castro-Martín, 2007; Castro-Martín y Rosero-Bixby, 2011; del Rey et al., 2015). Este estudio sólo contempla el comportamiento registrado en la reciente ola inmigratoria hasta 2007. Este recorte temporal nos permite analizar la fecundidad sin la interferencia generada por la crisis económica, la cual ha conllevado fuertes cambios en el comportamiento reproductivo de la población inmigrante con independencia del grado de integración o asimilación en la sociedad de destino.

La principal diferencia de la fecundidad inmigrante respecto a los estudios sobre la fecundidad en general deriva del propio hecho de migrar. El traslado y las implicaciones que conlleva afectan el comportamiento reproductivo de las mujeres inmigrantes, tanto en el momento anterior a la migración como inmediatamente posterior (Toulemon, 2004; Devolder y Bueno, 2011; del Rey y Grande, 2015). Esto requiere adoptar una visión 
dinámica de la fecundidad para poder separar el efecto de la migración del posible proceso de asimilación e integración a la sociedad receptora. De ahí, que adoptemos una perspectiva longitudinal que nos permite observar el nivel de fecundidad de las mujeres inmigrantes desde el mismo momento de llegada y analizar su evolución en la medida en que se prolonga su tiempo de residencia.

En primer lugar, en relación a la evolución del índice sintético de fecundidad entre 1998 y 2007 (Figura 1), se observa que en el inicio del boom migratorio los niveles de fecundidad de las mujeres inmigrantes y latinoamericanas eran mucho más altos que los de las mujeres españolas. Pero dicho nivel se ha ido asimilando al de la población nativa en la medida en que se ha consolidado el proceso migratorio. No obstante, este índice tradicional para el estudio de la fecundidad no toma en cuenta los diferentes momentos en el proceso de movilidad, es decir, no diferencia entre las mujeres que acaban de llegar de las mujeres que ya llevan un determinado tiempo en España, y por lo tanto, no toma en cuenta el impacto que el momento de la migración genera en la fecundidad.

Figura 1: Índice sintético de fecundidad en España según origen de la madre, 1997-2007

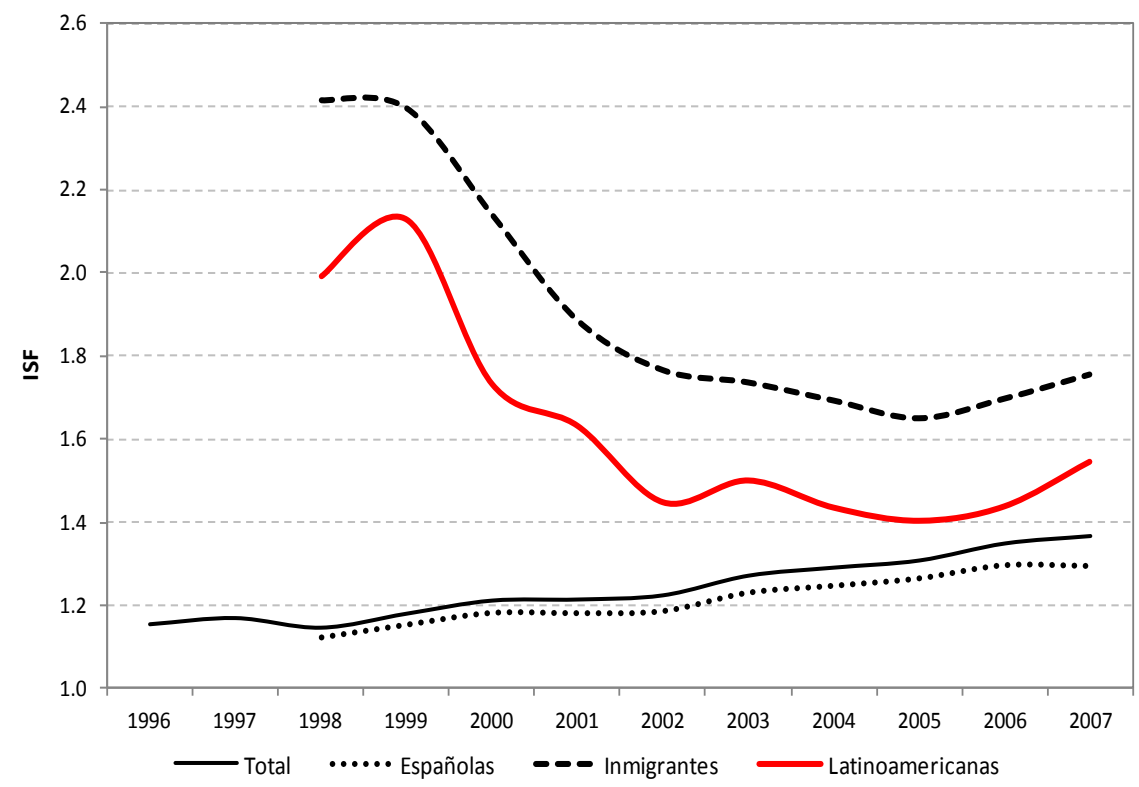

Fuente: elaboración propia a partir de Estadísticas del Movimiento Natural de la Población y del Padrón Municipal Continuo (Instituto Nacional de Estadística - INE). 
En segundo lugar, al considerar el momento de llegada (Figura 2), se obtiene un patrón de fecundidad muy diferente entre la población inmigrante. Los resultados muestran el impacto del momento de la migración en la fecundidad, tanto en el periodo inmediatamente anterior como en el posterior. Ahora bien, intentando aislar la perturbación que genera el desplazamiento, permanece la cuestión objeto de este estudio, esto es, si la fecundidad inmigrante tiende a asimilarse con la población española o por el contrario, tienden a reproducir la fecundidad de la sociedad de origen.

Figura 2: Índice sintético de fecundidad de las inmigrantes en España llegadas entre 1995 y 2007 en relación al año de llegada por lugar de procedencia

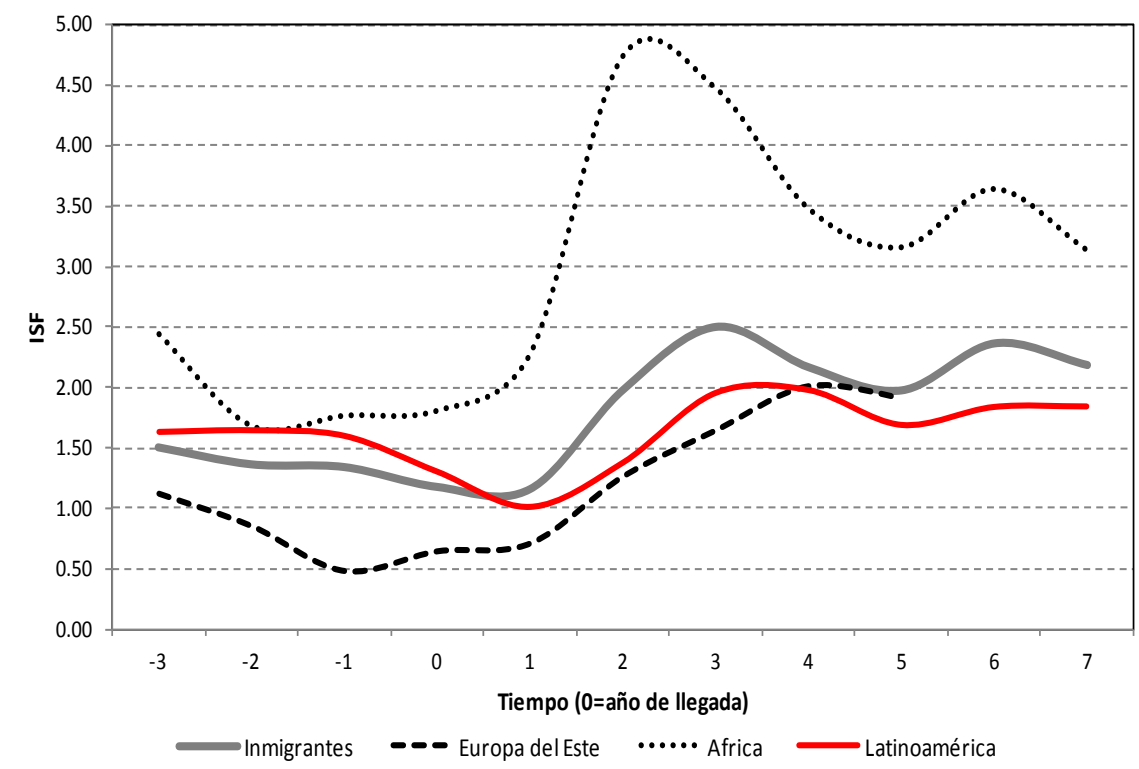

Fuente: elaboración propia a partir de la Encuesta Nacional de Inmigrantes de 2007 (INE).

\section{MARCO DE REFERENCIA: EL COMPORTAMIENTO REPRODUCTIVO DE LA POBLACIÓN INMIGRANTE}

La gran mayoría de trabajos sobre la fecundidad de la población inmigrante en la sociedad de destino, se centran exclusivamente en la población procedente de países menos desarrollados, la cual suele presentar un mayor nivel de fecundidad que la sociedad receptora (ver para el caso de España: Bledsoe et al., 2007; Roig-Vila y Castro-Martín, 2007). Este es el caso aquí planteado y dónde radica la importancia de conocer el comportamiento 
reproductivo, tanto por su transcendencia para el conjunto de la natalidad del país de destino (del Rey y Cebrián, 2010), como por el reflejo que tiene dicho comportamiento en medir el grado de integración en la sociedad receptora.

Desde la perspectiva demográfica, la fecundidad es resultado de la combinación de, por un lado, determinadas características socio-económicas de las mujeres/parejas (Coleman, 1994; Kahn, 1994; Milewski, 2007); $\mathrm{y}$, por otro lado, de la existencia de unos ideales y valores sobre la familia derivados del patrón cultural prevaleciente, a su vez resultado de la fase de transición demográfica en que se encuentra dicha sociedad (Bongaarts, 2003; Andersson, 2004; Roig-Vila y Castro-Martín, 2007; Sobotka, 2008). La migración implica de manera automática un cambio del patrón cultural de referencia en el comportamiento reproductivo, lo cual junto con los nuevos condicionamientos sociales y económicos, pueden conllevar un cambio en la fecundidad de las mujeres inmigrantes. Los trabajos que se han dedicado a estudiar la fecundidad de la población inmigrante en relación a la fecundidad de la sociedad de destino tienden a resaltar los siguientes comportamientos.

Desde hace décadas, la corriente mayoritaria en las investigaciones señala la existencia de un proceso de asimilación - adaptación según el cual los inmigrantes gradualmente ajustan su comportamiento reproductivo al del país de destino (Kahn, 1988 y 1994; Andersson, 2004; Parrado y Morgan, 2008).

Una segunda corriente señala que ciertos colectivos de inmigrantes tienden a mantener las normas y patrones reproductivos del país de origen (Abbasi-Shavazi y McDonald, 2002). El mantenimiento de la fecundidad de origen aparece muy ligado a migraciones familiares y a frecuentes contactos con el lugar de procedencia. Otros trabajos tratan de explicar esta falta de adaptación del patrón reproductivo a la sociedad de destino por la existencia de un proceso de selección entre los migrantes (Goldstein, 1973; Feliciano, 2005; Bledsoe et al., 2007; Sobotka, 2008) o por un proceso de asimilación segmentado debido a la exclusión y las dificultades para integrarse (Portes y Zhou, 1993).

Una tercera corriente ha constatado la existencia de un proceso de interrupción - ruptura en la fecundidad de la población inmigrante causado en general por el momento de migración y la separación de los esposos (Ford, 1990; Stephen y Bean, 1992; Carter, 2000; Toulemon, 2004; Kulu, 2005; Lindstrom y Giorguli, 2007; Milewski, 2007). Por lo tanto, la migración produce por sí misma un particular comportamiento reproductivo, antes, 
durante y con posterioridad al desplazamiento. Esto lleva a que a la hora de estudiar las pautas reproductivas y el grado de adaptación al patrón de la sociedad de destino sea necesario considerar el "momento de migración". Diversos trabajos han mostrado que la fecundidad de la población latinoamericana migrante en España se ve fuertemente alterada alrededor del momento del desplazamiento, tanto antes como después de migrar (Devolder y Bueno, 2011; del Rey y Grande, 2015). Sin embargo, un grave problema para analizar el efecto del "momento de la migración" es la falta de registros longitudinales con las historias reproductivas previas a la migración (Toulemon, 2004; Kulu, 2005; Roig-Vila y Castro-Martín, 2007; Parrado, 2011), de ahí la carencia de tales estudios. En este trabajo contamos con una fuente de datos que nos permite conocer la situación marital y reproductiva en el país de origen y considerar dichas situaciones en el comportamiento reproductivo en el país de destino.

A partir de lo anteriormente señalado, formulamos las siguientes hipótesis de trabajo:

- Hipótesis 1: en general consideramos la existencia de un proceso de asimilación para el conjunto de la población inmigrante objeto de estudio, si bien esperamos que dicho proceso se vea fuertemente afectado o interrumpido alrededor del momento de la migración.

- Hipótesis 2: la pervivencia o mantenimiento de los patrones de origen serán más persistentes en los inmigrantes procedentes de contextos en fases tempranas de transición demográfica y en los inmigrantes que llegan en fases avanzadas del ciclo de vida familiar.

- Hipótesis 3: esperamos, por el contrario, que los inmigrantes procedentes de contextos en fases avanzadas de transición demográfica y aquellos inmigrantes que llegan a edades más tempranas, y por lo tanto tienen una mayor socialización con los valores de la sociedad de destino, se producirá una más mayor asimilación en su fecundidad.

\section{FUENTE DE DATOS Y METOdOLOGÍA: UNA APROXIMACIÓN} LONGITUDINAL

El estudio del comportamiento reproductivo de las mujeres inmigrantes llevado a cabo en este trabajo se basa, fundamentalmente, en la Encuesta Nacional de Inmigrantes de 2007 (ENI-2007) del Instituto Nacional de Estadística (INE). De acuerdo con el objetivo propuesto se ha seleccionado una submuestra de 3157 casos - de los 15465 casos totales- correspon- 
dientes a las mujeres inmigrantes latinoamericanas y caribeñas que llegaron a España entre 1990 y 2007. Como han señalado, trabajos precedentes (Castro y Rosero-Bixby, 2011; Del Rey et al., 2015), la ENI-2007 presenta importantes ventajas para estudiar la interacción entre migración y fecundidad debido a que permite adoptar una perspectiva de análisis longitudinal mediante la reconstrucción de las historias completas de migración y nacimientos. Con estos datos longitudinales se puede observar la fecundidad de las mujeres antes y después de la migración.

Sin embargo, la ENI-2007 también presenta algunas limitaciones para el estudio del comportamiento reproductivo de la población inmigrante. Por un lado, la reconstrucción de la historia de nacimientos no permite trabajar con una medición del tiempo más precisa que los años, por ejemplo el mes de llegada sólo se registra para quienes lo hicieron con posterioridad a 2004. Por otro lado, no proporciona información detallada para reconstruir la historia laboral completa de los inmigrantes durante su residencia en España. Esto impide el uso de modelos longitudinales que pongan en relación el comportamiento reproductivo y la movilidad ocupacional, procesos mutuamente interdependientes.

En el análisis descriptivo del comportamiento reproductivo de la población inmigrante en España, además de la información extraída de la ENI-2007, se usan datos del Padrón Municipal Continuo y del Movimiento Natural de la Población (MNP) del INE. Los datos relativos a los patrones de fecundidad de la población en los países de origen se han obtenido de fuentes secundarias (CELADE, 2001 y CELADE, 2013) y de los censos de población de cada uno de los diferentes países seleccionados de América Latina y el Caribe.

En segundo lugar, el tratamiento de la información se ha desarrollado en dos fases. Primero, una descripción e interpretación de los indicadores clásicos de fecundidad, como son el índice sintético y las tasas específicas por edad, con la novedad de incorporar el momento de llegada. A continuación se han aplicado diferentes modelos de supervivencia en tiempo discreto para controlar las variables que pueden afectar el comportamiento reproductivo, tanto las que tienen que ver con las características personales y familiares, como con el lugar de origen.

Los modelos de supervivencia permiten analizar la ocurrencia de un evento a lo largo del tiempo, es decir, el riesgo relativo o probabilidad condicional del nacimiento del primer hijo después de la migración. La duración de la transición se ha calculado tomando en cuenta el año de llegada y el año del nacimiento del primer hijo en la sociedad de destino. De 
esta manera, se ha trasformado la base de datos de la ENI-2007 en registros correspondientes a años-mujer, pasando a trabajar con 37,884 registros correspondientes a las mujeres de origen latinoamericano y caribeño que llegaron a España entre 1990 y 2007 y que se encontraban en edad fértil durante el periodo de estudio. La variable dependiente es el tiempo transcurrido desde el momento de la llegada hasta tener el primer hijo en la sociedad de destino o bien hasta residir 6 años en España sin haber tenido ningún hijo. El periodo de observación de la trayectoria reproductiva se ha limitado a los primeros seis años tras la migración para no sobre-ponderar el riesgo del nacimiento del primer hijo de las inmigrantes llegadas al inicio del periodo de observación y, por el contrario, sub-ponderar a las inmigrantes llegadas más recientemente. Esto es importante debido a que 47.9 por ciento de las inmigrantes latinoamericanas llegaron a España a partir de 2001, es decir que casi la mitad de los casos no superarían los seis años de residencia.

\section{RESULTADOS Y DISCUSIÓN: DETERMINANTES DE LA FECUNDIDAD}

El análisis de la fecundidad de la población inmigrante en las sociedades de destino se articula en torno a la disyuntiva entre la adaptación al patrón de la sociedad de acogida y el mantenimiento de las pautas reproductivas de la sociedad de partida. Esto requiere, como se ha señalado, prestar atención a varios aspectos: unos tienen que ver con la fecundidad en general, como son los referentes culturales o ciertas características socioeconómicas, mientras otros con el propio impacto del momento de la migración. Se empezará por poner en relación la fecundidad migrante con la de la población de origen y destino.

\section{La fecundidad migrante en relación a la fecundidad de origen y destino}

En primer lugar analizamos la fecundidad inmigrante en relación a la existente en los países de origen y en España, considerando tanto el indicador de intensidad como de calendario a través de las tasas por edad.

La Tabla 1 muestra la evolución del índice sintético de fecundidad (ISF) para distintos países de América Latina, así como el de las mujeres inmigrantes en España en el año 2007. Lo primero que se observa en este indicador transversal es que las mujeres latinoamericanas en España presentan un nivel de fecundidad muy inferior al de sus países de procedencia, aunque ligeramente superior al de España (1.37). Frente a los 2.37 hijos por mujer para el total de América Latina y el Caribe, las inmigrantes en España tienen únicamente 1.56 hijos por mujer. 
Tabla 1: Evolución de los índices sintéticos de fecundidad en América Latina y países seleccionados entre 1955-2010 y de las inmigrantes latinoamericanas en España en 2007

\begin{tabular}{|c|c|c|c|c|c|c|c|}
\hline & \multicolumn{6}{|c|}{ Evolución del ISF en América Latina } & \multirow{2}{*}{$\begin{array}{c}\begin{array}{c}\text { ISF Inmigrantes } \\
\text { en España }\end{array} \\
2007\end{array}$} \\
\hline & $\begin{array}{c}1955- \\
1960\end{array}$ & $\begin{array}{l}1965- \\
1970\end{array}$ & $\begin{array}{c}1975- \\
1980\end{array}$ & $\begin{array}{l}1985- \\
1990\end{array}$ & $\begin{array}{l}1995- \\
2000\end{array}$ & $\begin{array}{l}2005- \\
2010\end{array}$ & \\
\hline América Latina & 5.94 & 5.57 & 4.50 & 3.42 & 2.74 & 2.30 & 1.56 \\
\hline Cuba & 3.70 & 4.30 & 2.15 & 1.85 & 1.61 & 1.49 & 1.20 \\
\hline Honduras & 7.50 & 7.42 & 6.60 & 5.37 & 4.30 & 3.31 & 1.47 \\
\hline México & 6.80 & 6.75 & 5.25 & 3.63 & 2.67 & 2.37 & 1.10 \\
\hline Nicaragua & 7.50 & 6.95 & 6.35 & 5.00 & 3.60 & 2.76 & 1.27 \\
\hline R. Dominicana & 7.64 & 6.65 & 4.76 & 3.47 & 3.05 & 2.67 & 1.73 \\
\hline \multicolumn{7}{|c|}{ Región: México, Centroamérica y Caribe } & 1.46 \\
\hline Bolivia & 6.75 & $6.56^{\circ}$ & 5.80 & 5.00 & 4.32 & 3.50 & 1.90 \\
\hline Colombia & 6.76 & 6.18 & 4.34 & 3.17 & 2.70 & 2.45 & 1.46 \\
\hline Ecuador & 6.70 & 6.50 & 5.40 & 4.00 & 3.10 & 2.75 & 1.74 \\
\hline Perú & 6.85 & 6.56 & 5.38 & 4.10 & 3.10 & 2.43 & 1.52 \\
\hline \multicolumn{7}{|c|}{ Región: Países Andinos } & 1.70 \\
\hline Argentina & 3.13 & 3.05 & 3.44 & 3.05 & 2.63 & 2.25 & 1.32 \\
\hline Brasil & 6.15 & 5.38 & 4.31 & 3.10 & 2.45 & 1.95 & 1.43 \\
\hline Chile & 5.49 & 4.44 & 2.80 & 2.65 & 2.21 & 1.90 & 1.26 \\
\hline Paraguay & 6.50 & 6.15 & 5.20 & 4.77 & 3.88 & 3.08 & 1.47 \\
\hline Uruguay & 2.83 & 2.80 & 2.89 & 2.53 & 2.30 & 2.12 & 1.32 \\
\hline Venezuela & 6.46 & 5.90 & 4.47 & 3.65 & 2.94 & 2.55 & 1.22 \\
\hline \multicolumn{7}{|c|}{ Región: Resto de Sudamérica } & 1.35 \\
\hline
\end{tabular}

Fuente: CELADE (2013) para la evolución en América Latina, y Movimiento Natural de la Población y Padrón Municipal Continuo (INE) para los inmigrantes en España.

Aunque se trata de un indicador coyuntural, las diferencias observadas a primera vista permiten señalar diferentes hipótesis: una, la existencia de un proceso de adaptación al comportamiento reproductivo en destino; la segunda es un proceso de selección en las migraciones, es decir, las inmigrantes que llegan a España desde Latinoamérica no serían representativas del conjunto de la población; tres, la combinación de las dos hipótesis anteriormente mencionadas. A pesar de la dificultad de medir el impacto de la selectividad, debido a la limitación de las fuentes de datos, se puede platear la hipótesis de que la menor fecundidad pudiera estar relacionada con el origen socioeconómico dentro de las sociedades de origen. Algo a tener 
en cuenta dadas las significativas diferencias en la fecundidad según nivel socioeconómico y las diferencias entre zonas rurales y urbanas en América Latina (Rosero-Bixby, 2004; Grande, 2012).

Además, se observa que a mayor intensidad de la fecundidad en origen, mayor es la intensidad de la fecundidad entre las mujeres que han emigrado a España, por lo que se aprecia un claro efecto del patrón de origen. Por otro lado, no se puede obviar que el ISF es una medida en un momento determinado del tiempo, por lo que a la hora de analizar la fecundidad de las inmigrantes se puede ver distorsionado por un posible efecto llegada como se mostró en la Figura 2. En otras palabras, al medir la intensidad de la fecundidad en 2007, muchas de las mujeres latinoamericanas aún recién llegadas tienden a disminuir su fecundidad de manera temporal según lo propuesto por la hipótesis de la interrupción. En especial, las procedentes de aquellos países que fueron los últimos en incorporarse al boom inmigratorio hacia España. Esto puede explicar los ISF relativamente bajos en comparación con sus países de origen de las mujeres procedentes de Honduras, Nicaragua o Paraguay, cuyos contingentes empezaron a llegar a España de forma significativa a partir de 2006. Por el contrario, las dominicanas y bolivianas con un ISF más alto podrían encontrarse en 2007 en la fase de recuperación de la fecundidad, tras la reducción sufrida alrededor del momento de la migración. Sobre esto volveremos más adelante para analizar el efecto del momento de llegada sobre la fecundidad.

En la Figura 3 se presentan para los países seleccionados y el total regional, las tasas específicas de fecundidad (TEF) por edades de las mujeres en los países de origen, las inmigrantes en España en 2007 y las nativas españolas en 2007, lo cual nos permite visualizar los calendarios reproductivos. La población de América Latina se caracteriza por presentar un patrón reproductivo concentrado en edades tempranas, muy influenciado por la edad de iniciación sexual y por la reducción de la tenencia de hijos en edades superiores a los 30 años (Chackiel, 2004; Di Cesare, 2007). Esa distribución contrasta con el calendario reproductivo notablemente retrasado de las nativas españolas, quienes tienen la TEF más elevada entre los 30 y los 34 años.

Respecto al comportamiento de la población migrante, los resultados muestran importantes diferencias según el país y la región de origen, lo que viene a confirmar que sus pautas reproductivas son muy heterogéneas. A grandes rasgos se puede afirmar que las inmigrantes latinoamericanas en España mantienen un calendario reproductivo más temprano que las nativas, pero a la vez menos concentrado en edades jóvenes que el de sus países de origen. No obstante se observan ciertas particularidades reseñables. 
Figura 3: Tasas específicas de fecundidad por edad en América Latina y países en 2005-2010 y de las inmigrantes latinoamericanas en España en 2007
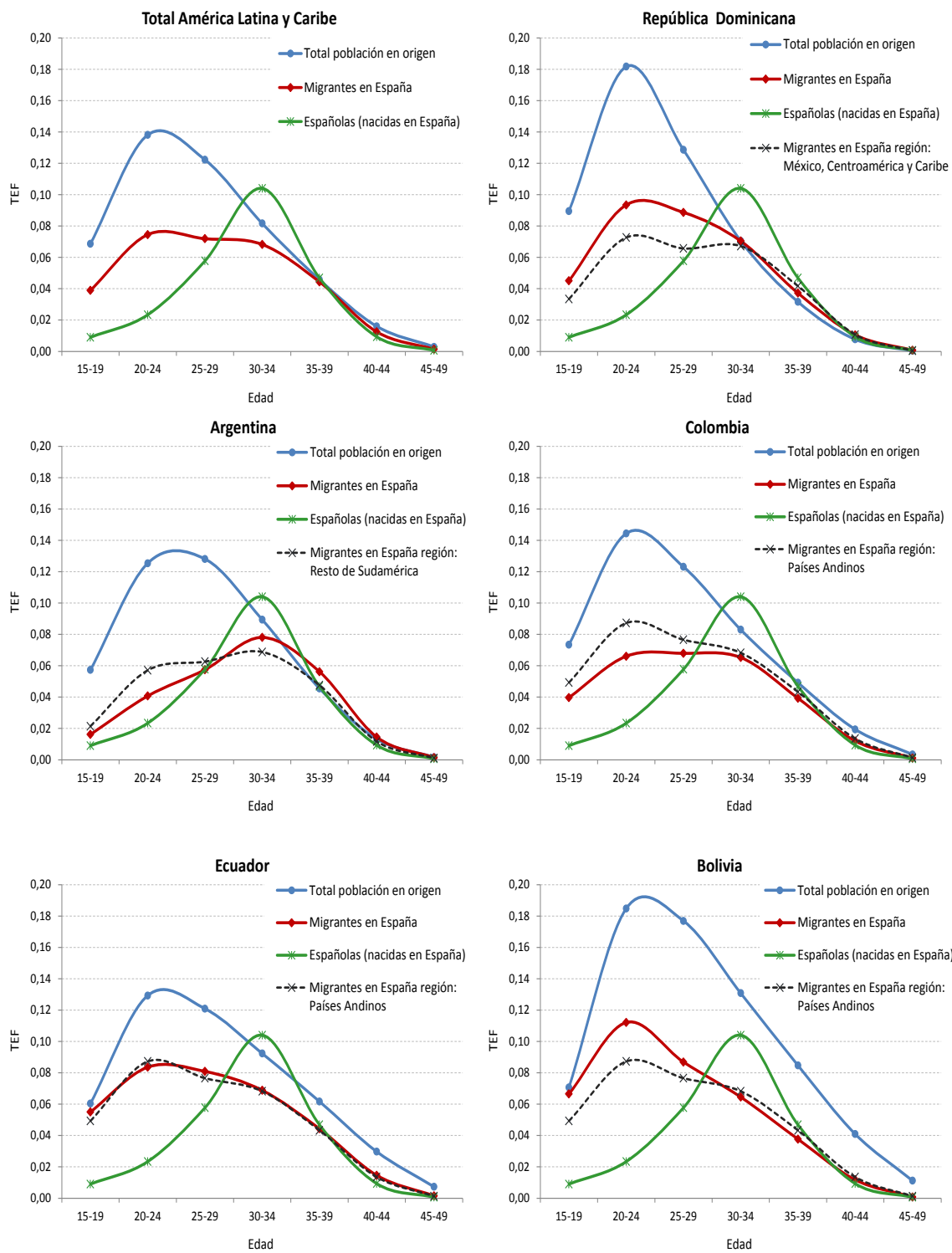

Nota: Los datos proporcionados por CELADE (2001) para las tasas específicas por edades en América Latina deben ser observadas con cautela ya que las cifras proporcionadas son estimaciones; aunque el margen de error no es excesivo por la cercanía temporal de la estimación lo cual permite el análisis comparativo.

Fuente: CELADE (2001) para América Latina y Caribe, y Movimiento Natural de la Población y Padrón Municipal Continuo (INE) para las españolas y los inmigrantes en España. 
Primero, destacan especialmente las inmigrantes dominicanas, ecuatorianas y bolivianas que mantienen una mayor fecundidad en edades jóvenes respecto al comportamiento de las españolas. En cambio, las argentinas - y en general el resto de sudamericanas - concentran su calendario reproductivo a partir de los 30 años con un comportamiento más similar a las nativas, lo cual se explica por encontrarse estos países en fases más avanzadas de la transición demográfica. En suma, el mantenimiento de esa tendencia corroboraría el efecto de las pautas culturales en el comportamiento reproductivo de las mujeres inmigrantes.

Segundo, pese al mantenimiento de un calendario reproductivo temprano éste está retrasado en relación al del total de mujeres en origen. En todos los países seleccionados las inmigrantes tienen un calendario reproductivo más retrasado que sus compatriotas en el país de origen. Por lo tanto, junto a la menor intensidad, la fecundidad de las inmigrantes latinoamericanas respecto a sus países de origen se concentra más entre los 30-39 años y menos en edades tempranas.

\section{El efecto del momento de llegada en la fecundidad migrante}

Una de las principales ventajas de disponer de datos retrospectivos es poder conocer la fecundidad de las inmigrantes antes y después de la migración, es decir adoptando una perspectiva longitudinal. En primer lugar, usando las TEF se estudia el efecto de la edad a la llegada a España sobre el calendario reproductivo del conjunto de las mujeres latinoamericanas y en segundo lugar, se analiza las mismas tasas para las tres diferentes regiones consideradas.

La Figura 4 muestra las diferencias en el calendario reproductivo según la edad a la llegada. Salvo para las mujeres llegadas con 35 años o más, para el resto de grupos no se dispone de la evolución completa de su calendario reproductivo dado que sólo se estudia las inmigrantes llegadas entre 1990-2007 y, por tanto, en 2007 no habían completado aún su periodo fértil. A pesar de esta censura en las observaciones, se aprecian tres tendencias fundamentales.

En primer lugar, las mujeres que migraron a España casi al final de su ciclo reproductivo (con 35 años o más) muestran un calendario temprano, con altas tasas de fecundidad a edades jóvenes, es decir, un patrón similar al de sus países de origen. En este caso no se aprecia un efecto del movimiento migratorio, puesto que las tasas de fecundidad siguen disminuyendo tras la llegada por la fase del ciclo de vida en la que se produce la migración. 
Figura 4: Tasas específicas de fecundidad por edad, según grupo de edad en el momento de la llegada. Mujeres latinoamericanas llegadas entre 1990-2007

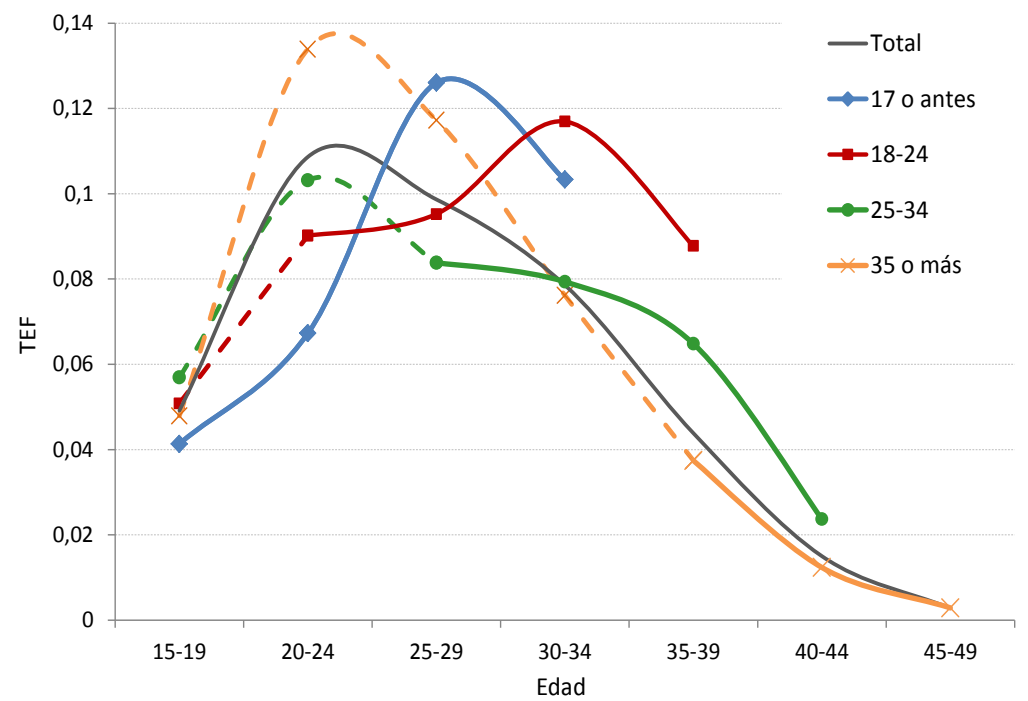

Nota: La línea con guiones indica el periodo previo a la migración y la línea sólida indica el periodo posterior a la migración de forma aproximada para cada grupo de edad según la edad en el momento de la llegada. Fuente: elaboración propia a partir de ENI 2007.

En segundo lugar, en las mujeres que llegaron en pleno periodo reproductivo, sí se observa un efecto de la migración en el calendario reproductivo. Por un lado, se da un efecto contención de la fecundidad previa a emigrar, que se refleja en las menores tasas de fecundidad a edades jóvenes. Así, las latinoamericanas que llegaron entre los 18 y 24 años presentan una baja fecundidad en las edades en que tuvo lugar el movimiento migratorio, mientras que para las mujeres que llegaron a España entre 25 y 34 años hay un claro descenso de la fecundidad en estas edades. Por otro lado, se aprecia un efecto repunte o de recuperación de la fecundidad posterior a la migración. Para las que llegaron entre los 18 y los 24 años, su edad cúspide para ser madres se sitúa entre los 30 y los 34 años. En cambio, para las mujeres que llegaron entre los 25 y los 34 años se observa una cierta prolongación de la fecundidad en edades avanzadas respecto a las que llegaron con 35 años o más.

En tercer lugar, las latinoamericanas que llegaron a España antes o al inicio de su periodo reproductivo, es decir, siendo menores de edad, muestran inicialmente un calendario más tardío, concentrando su fecundidad a partir de los 25 años. En este caso la migración no parece interferir en las 
pautas reproductivas y aunque se trata de observaciones incompletas al no haber terminado su periodo reproductivo, se aprecia un comportamiento bastante similar al de la población de destino. Estos resultados se pueden interpretar como una señal de adaptación a las pautas de destino, ya sea por asimilación debido a su escasa socialización en origen o por verse afectadas por una similar estructura de oportunidades que las nativas que condiciona el retraso de la fecundidad para las que llegaron más jóvenes.

Al separar por la región de origen (Figura 5), se aprecian de nuevo importantes diferencias según edad a la llegada y región de origen. Las inmigrantes andinas destacan frente a los otros colectivos por su mayor nivel de fecundidad y su calendario más temprano en todos los grupos de edad a la llegada, lo cual es un rasgo característico de las pautas reproductivas en sus países de origen. Lo más llamativo es la mayor fecundidad a edades tempranas también entre las andinas que llegaron antes de iniciar su ciclo reproductivo (llegadas antes de los 18 años). Estos resultados apuntan a una menor tendencia de este colectivo a la adaptación. Sin embargo, es importante considerar paralelamente las diferentes características sociodemográficas de la migración andina frente a los otros colectivos, lo cual será controlado más adelante a través del uso de modelos multivariables.

Las mujeres que llegaron en pleno ciclo reproductivo (18-24 y 25-34 años), al realizar el análisis según la región de origen, presentan un comportamiento claramente afectado por el movimiento migratorio: con un descenso de la fecundidad coincidiendo con el momento de la migración $\mathrm{y}$ un posterior repunte tras unos primeros años de residencia en destino. Se comprueba así la existencia de un efecto interrupción que provoca un cierto retraso del calendario reproductivo.

\section{Determinantes del comportamiento reproductivo en destino}

Por último, se han realizado diversas regresiones en tiempo discreto que permiten conocer los determinantes del riesgo o probabilidad condicional de tener el primer hijo en España a lo largo de los seis primeros años tras la llegada. Se han estimado tres modelos que toman en cuenta diferentes variables independientes que pueden estar afectando la probabilidad de tener este primer hijo (Tabla 2). El primer modelo incluye las características socio-demográficas y relativas a la migración. El segundo modelo incorpora también las condiciones familiares en el momento de la llegada y su evolución en cada momento del periodo de estudio. El tercer modelo incorpora además la situación ocupacional inicial en España y antes de migrar en el país de origen. 

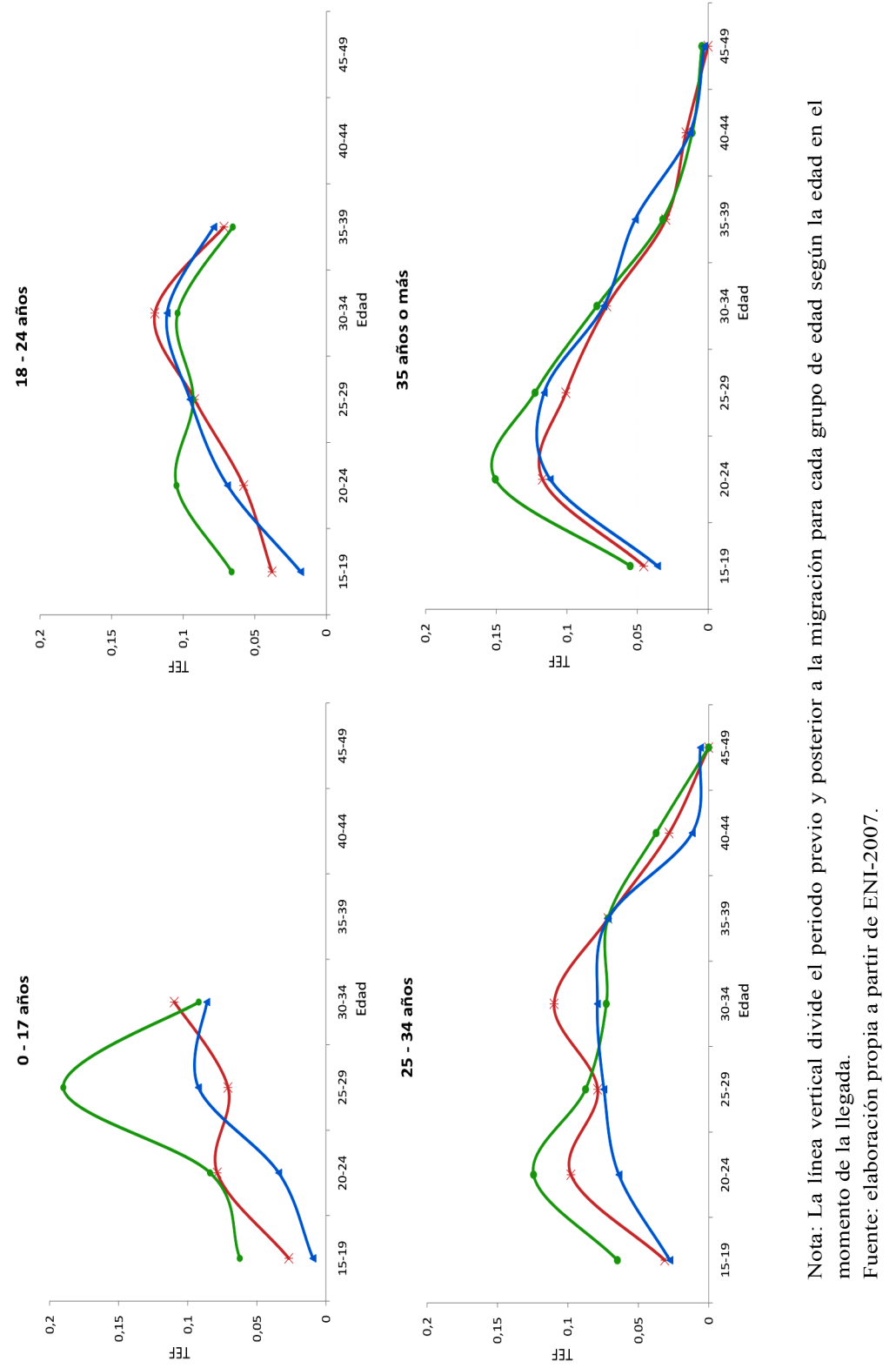
La región de procedencia tiene una incidencia significativa en los tres modelos controlando terceras variables. El riesgo de tener el primer hijo en la sociedad de destino disminuye para los colectivos de mujeres no andinas, siendo las andinas la categoría de referencia. Estas diferencias son más significativas cuando se controla la situación familiar y ocupacional en los modelos 2 y 3 (Tabla 2). Estos resultados constatan la existencia de un comportamiento reproductivo diferente según el lugar de procedencia, lo cual remite fundamentalmente al mayor mantenimiento de las normas y patrones culturales y reproductivos del país de origen por parte de las andinas. Tampoco es descartable la existencia de diferentes procesos de selección e incluso el mayor componente familiar en la migración andina (Grande y del Rey, 2012).

Respecto a las características de la migración, el año y la edad a la llegada, son estadísticamente significativos en los tres modelos. Por un lado, a mayor edad en el momento de la llegada, menor es el riesgo de tener un primer hijo en España para las inmigrantes en edad fértil. Las inmigrantes que llegaron al final de su ciclo reproductivo (35 años o más) son menos propensas a tener un primer hijo en España, siendo este efecto altamente significativo. Las mujeres que llegaron al inicio de su ciclo reproductivo (15-24 años) tiene ligeramente más riesgo de tener un primer hijo que las que lo hicieron entre los 25 y 34 años que es la categoría de referencia, pero esta diferencia desaparece al controlar por la situación familiar (modelo 2). Por otro lado, la probabilidad condicional de tener el primer hijo disminuye significativamente cuanto más reciente es la llegada, de forma lógica dado el menor tiempo en España para observar su comportamiento reproductivo y también debido a la reducción que conlleva el momento de la migración en la fecundidad en los primeros años de estancia, como se ha apuntado en el patrón general (Figura 2). Aquí el año de llegada resulta interesante como variable de control que evita una distorsión de los resultados.

El nivel de estudios no tiene un efecto relevante, salvo cuando se incorporan las variables sobre la situación ocupacional (modelo 3). En ese caso, de acuerdo con lo apuntado en la literatura, las inmigrantes con mayor nivel de estudios tienen menor probabilidad condicional de tener el primer hijo en España.

Las otras variables explicativas relacionadas con el movimiento migratorio, nacionalidad a la llegada y motivos de la migración, no ejercen una influencia significativa en ninguno de los tres modelos, al menos durante los primeros seis años de residencia. 
Tabla 2a: Probabilidad condicional de tener el primer hijo después de emigrar. Análisis de eventos en tiempo discreto para las mujeres inmigrantes latinoamericanas en edad fértil (15-49) llegadas a España entre 1990 y 2007

\begin{tabular}{|c|c|c|c|c|}
\hline & & & Modelo 1 & \\
\hline & & $\begin{array}{l}\text { Odds } \\
\text { Ratio }\end{array}$ & $\begin{array}{l}\text { Robust } \\
\text { Std. Err }\end{array}$ & Sig. \\
\hline Región de & $\begin{array}{l}\text { México, Centroamérica y } \\
\text { Caribe }\end{array}$ & 0.667 & 0.090 & $* *$ \\
\hline origen & Andinos (ref.) & 1 & & \\
\hline & Resto de Sudamérica & 0.813 & 0.084 & * \\
\hline Año de llegada & & 0.923 & 0.009 & $* * *$ \\
\hline & $15-24$ & 1.133 & 0.101 & $* *$ \\
\hline Edad al llegar & $25-34$ (ref.) & 1 & & \\
\hline & $35-49$ & 0.190 & 0.033 & $* * *$ \\
\hline & Primaria o menos & 0.973 & 0.114 & \\
\hline estudios & Secundaria (ref.) & 1 & & \\
\hline & Superiores & 0.878 & 0.095 & \\
\hline Nacionalidad & No (ref.) & 1 & & \\
\hline $\begin{array}{l}\text { espanola antes } \\
\text { de migrar }\end{array}$ & Sí & 0.914 & 0.233 & \\
\hline & No económicos (re.) & 1 & & \\
\hline & Económicos & 0.900 & 0.087 & \\
\hline migración & $\begin{array}{l}\text { No reunificación familiar } \\
\text { (ref.) }\end{array}$ & 1 & & \\
\hline & Reunificación familiar & 1.078 & 0.110 & \\
\hline & Sin hijos (ref.) & & & \\
\hline $\begin{array}{l}\text { Hijos antes de } \\
\text { migrar }\end{array}$ & 1 hijo & & & \\
\hline & 2 o más hijos & & & \\
\hline Estado civil y & Solteras & & & \\
\hline convivencia a & Casadas y conviven con el & tyuge & & \\
\hline lo largo del & Casadas y no conviven cor & cónyuge & & \\
\hline periodo & Separadas, viudas & & & \\
\hline & Endógamo o connacional & & & \\
\hline (sólo casadas) & Mixto con español (ref.) & & & \\
\hline & Mixto tercer país & & & \\
\hline & Alto industrial y servicios & & & \\
\hline Ocupacion & Medio industrial y servicic & ef.) & & \\
\hline España & Bajo industrial y servicios & & & \\
\hline & Nunca trabajó en España & & & \\
\hline & Alto industrial y servicios & & & \\
\hline Situación y & Medio industrial y servicic & ef.) & & \\
\hline ocupación en & Bajo industrial y servicios & & & \\
\hline origen antes & Desempleo & & & \\
\hline de migrar & Estudios & & & \\
\hline & Tareas domésticas & & & \\
\hline & $\mathrm{N}$ & 13.586 & & \\
\hline & $\begin{array}{l}\text { Ajustado por clusters } \\
\text { (individuos) }\end{array}$ & 2.621 & & \\
\hline & Log likelihood & -2416.116 & & \\
\hline & Wald chi2 & 187.20 & & \\
\hline & Prob $>$ chi 2 & 0.0000 & & \\
\hline & Pseudo R2 & 0.049 & & \\
\hline
\end{tabular}


Tabla 2b: Probabilidad condicional de tener el primer hijo después de emigrar. Análisis de eventos en tiempo discreto para las mujeres inmigrantes latinoamericanas en edad fértil (15-49) llegadas a España entre 1990 y 2007

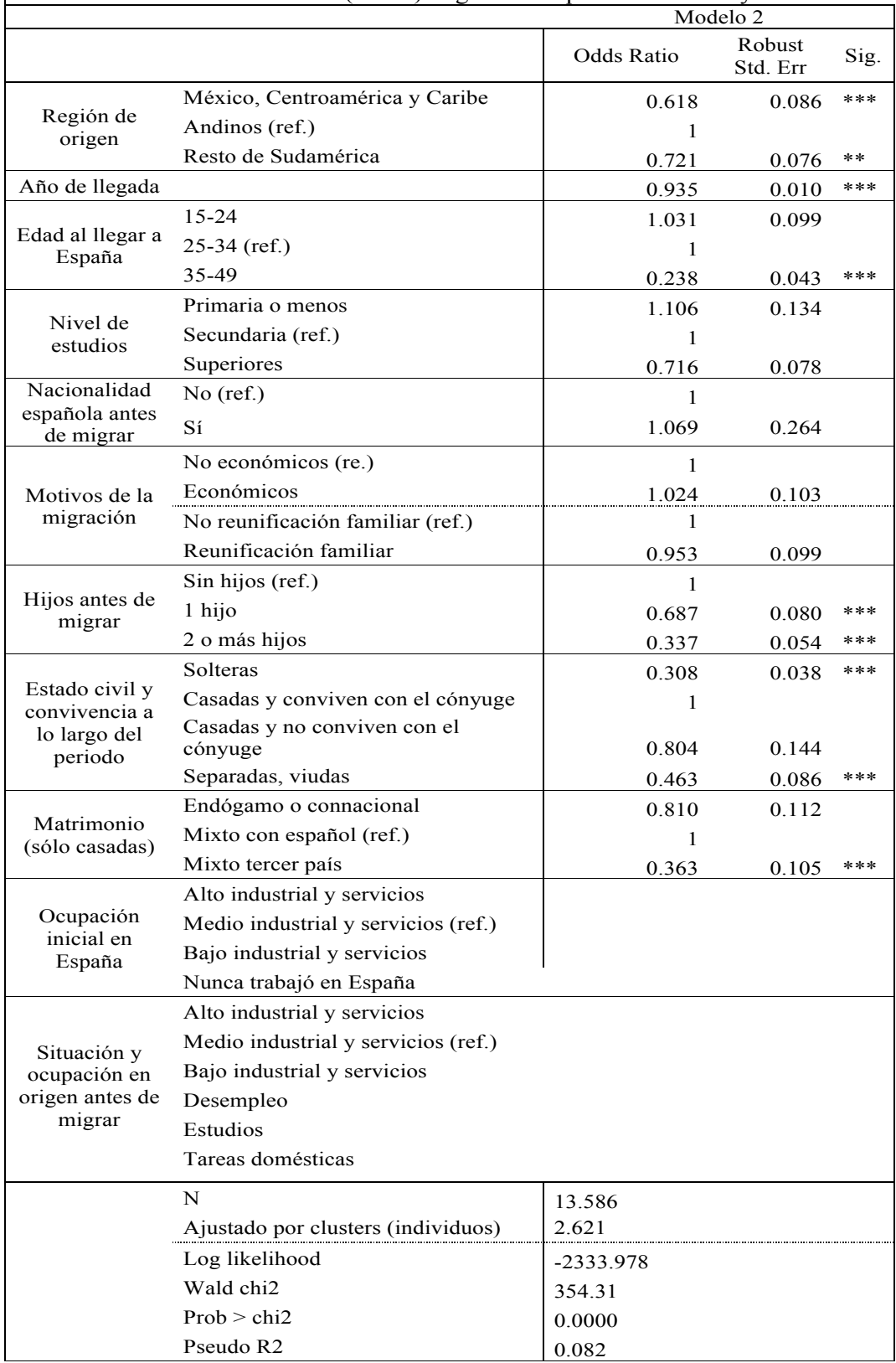

$* * *$ Significativo al 99.9\% $(\mathrm{P}<0.001) / * *$ Significativo al $99 \%(\mathrm{P}<0.01) / *$ Significativo al $95 \%(\mathrm{P}<0.05) / \bullet$ Significativo al $90 \%(\mathrm{P}<0.10)$

Fuente: elaboración propia a partir de ENI 2007. 
Tabla 2c: Probabilidad condicional de tener el primer hijo después de emigrar. Análisis de eventos en tiempo discreto para las mujeres inmigrantes latinoamericanas en edad fértil (15-49) llegadas a España entre 1990 y 2007

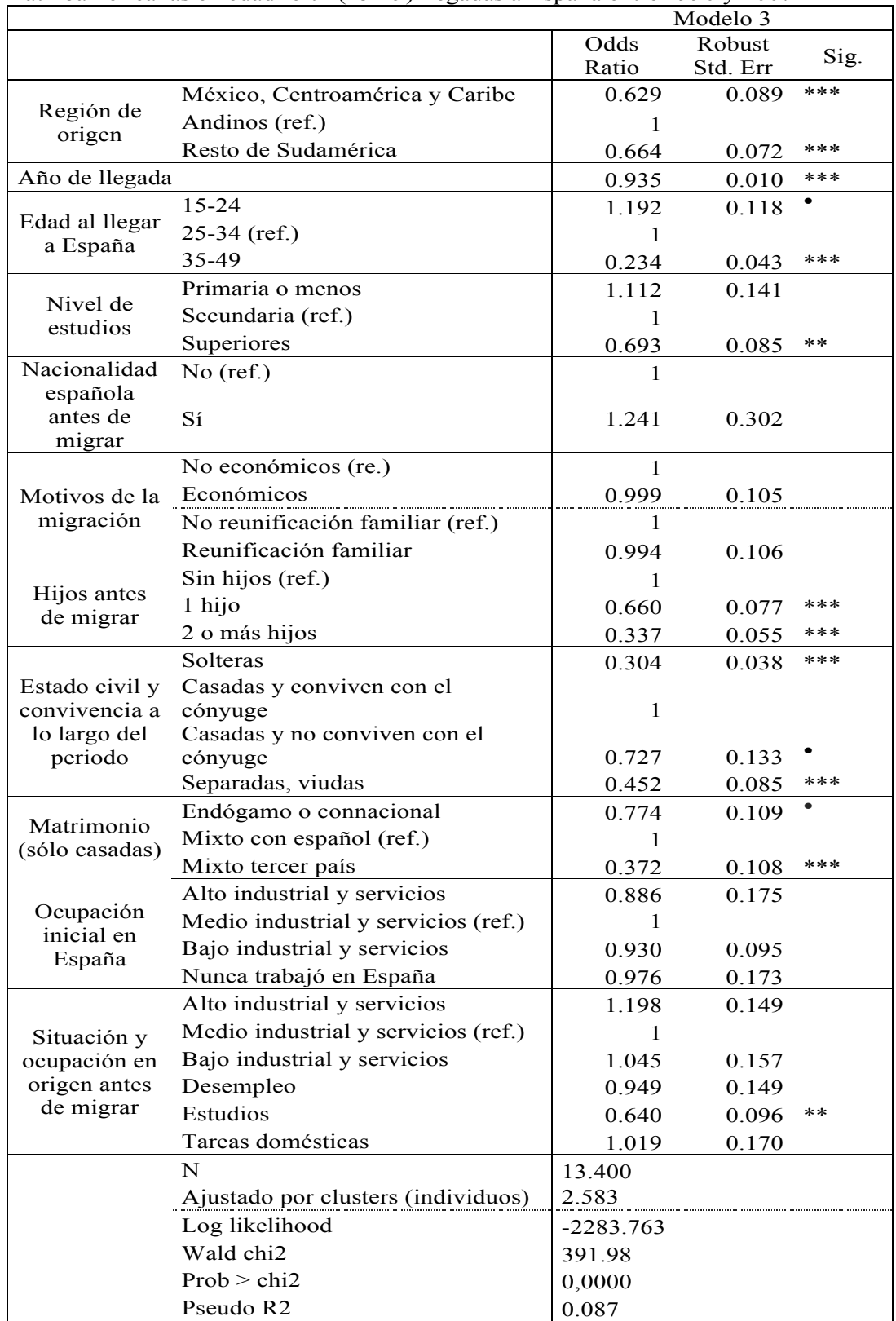

$* * *$ Significativo al $99.9 \%(\mathrm{P}<0.001) / * *$ Significativo al $99 \%(\mathrm{P}<0.01) / *$ Significativo al $95 \%(\mathrm{P}<0.05) / \bullet$ Significativo al $90 \%(\mathrm{P}<0.10)$

Fuente: elaboración propia a partir de ENI 2007. 
El nulo efecto de tener la nacionalidad española en el momento de la llegada puede deberse a que la condición legal no es una condición sine qua non que garantice mejores condiciones que favorezcan formar una familia en la sociedad de destino, al menos para la reciente migración latinoamericana. Tampoco se puede concluir que tener un hijo en la sociedad de destino sea menos "prioritario" entre aquellas con objetivos económicos que entre las que migran por motivos familiares, como han mostrado otros trabajos para el total de inmigrantes en España (Castro y Rosero-Bixby, 2011; Del Rey et al., 2015).

Las características familiares previas y su evolución durante los seis primeros años de residencia ejercen un efecto muy significativo sobre el riesgo de tener el primer hijo en España, efecto que se mantiene al incorporar las variables sobre la situación ocupacional. En este tercer modelo, las mujeres que han tenido un hijo antes de migrar reducen su probabilidad condicional de ser madres en España (34 por ciento) con respecto a quienes llegan sin haber tenido hijos, reducción que alcanza 66 por ciento en las mujeres que habían tenido dos o más hijos previos.

El estado civil y la convivencia con el cónyuge a la llegada igualmente ejercen un importante efecto en la probabilidad de tener un hijo. Frente a las mujeres casada y que conviven con el cónyuge en España, las inmigrantes latinoamericanas que no están en unión tienen una probabilidad muy inferior de tener un hijo en los primeros seis años. Así, en ambos modelos, las solteras tienen 70 por ciento menos de probabilidad de tener un hijo, y las separadas y viudas su probabilidad es inferior a 50 por ciento respecto a las casadas que viven con el cónyuge. Por su parte, las casadas que no conviven con el cónyuge tienen también menos riesgo de tener un hijo en España respecto a las que sí conviven o transitan a esta situación mediante la reagrupación del marido. Sin embargo, esta última diferencia sólo es significativa al controlar por la situación ocupacional.

El tipo de matrimonio de las casadas a lo largo del periodo de estudio también ejerce un interesante efecto en los modelos. Respecto a las casadas con un español, las que tienen un matrimonio mixto con un cónyuge de un tercer país tienen 62 por ciento menos de probabilidad de tener un hijo. Sin embargo, la diferencia entre matrimonio mixto con español y matrimonio con un connacional no es especialmente fuerte (sólo en el modelo 3), apuntando a una menor probabilidad de ser madres durante los primeros seis años de aquellas mujeres con uniones endogámicas respecto a las uniones mixtas con un español. 
En suma, se comprueba la importancia de la situación reproductiva previa y del estado marital como factores que condicionan su fecundidad en el lugar de destino. Primero, la importancia de la situación reproductiva previa vinculada a la interrelación entre fecundidad y migración. Segundo, los resultados apuntan a que la situación familiar y el estado civil guardan una estrecha relación o incluso condicionan los motivos de la migración, y de ahí la falta de significatividad estadística de estos últimos. Tercero, la influencia del asentamiento familiar como factor clave que favorece la fecundidad en destino. En este sentido, los resultados concuerdan con la perspectiva de la Nueva Economía de las Migraciones Laborales (NEML) que expone que los movimientos migratorios internacionales son, fundamentalmente, consecuencia de las dinámicas y estrategias familiares (Stark, 1991).

Finalmente, las variables sobre la situación ocupacional inicial y en el país de origen antes de migrar no tienen un efecto importante en el riesgo de tener el primer hijo en España en los seis años iniciales de residencia. Tan sólo las mujeres que antes de migrar se encontraban estudiando en su país de origen muestran una probabilidad significativamente inferior de ser madres en España. Esto se explicaría por el diferente tipo de migración de estas mujeres: buena parte de ellas son adolescentes que migraron junto a algún familiar o se trasladaron a España para continuar sus estudios. Este diferente tipo de migración favorecería su mayor adaptación a las pautas de fecundidad en destino o estar afectadas por una misma estructura de oportunidades que las nativas, lo que disminuye la intensidad de su fecundidad y retrasa su calendario. Este hallazgo, la importancia del tipo de migración y la edad a la llegada en la fecundidad de las inmigrantes latinoamericanas en España, concuerda con hallazgos en la misma línea, por ejemplo, para el caso de Italia (Mussino y Strozza, 2012).

\section{Conclusiones}

El objetivo general de este trabajo ha sido analizar las pautas de fecundidad de las mujeres inmigrantes latinoamericanas y caribeñas llegadas a España entre 1990 y 2007 desde la perspectiva de su integración en la sociedad de destino. Los resultados han mostrado un panorama complejo y diverso del patrón reproductivo desde las diferentes perspectivas estudiadas, reflejo de la propia heterogeneidad de la migración latinoamericana a España.

En primer lugar, la visión comparada respecto al total de mujeres en los países de origen y con respecto a la sociedad de destino ha confirmado la existencia de una fuerte interacción entre fecundidad y migración. El co- 
lectivo migrante presenta valores particulares tanto en su calendario como en su intensidad de la fecundidad que difieren de los valores de la sociedad de origen y de destino.

Sin embargo, los resultados también han mostrado la influencia de las pautas culturales y de la socialización en origen en el comportamiento reproductivo de las migrantes. Así, las andinas tienen un nivel de fecundidad mayor a los otros colectivos, un calendario reproductivo menos retrasado y una mayor probabilidad de ser madres en los primeros años en la sociedad de destino. Por su parte, el resto de mujeres sudamericanas muestra un comportamiento más similar al de las nativas, con una menor fecundidad y un calendario más retrasado. En este sentido, la mayor similitud entre origen y destino en estas últimas en relación a las fases de la transición demográfica explicaría la menor probabilidad de tener hijos en destino.

En segundo lugar, los hallazgos apuntan a una importante interacción entre fecundidad y migración también en relación al efecto del momento de la llegada. La fecundidad de las migrantes latinoamericanas desciende en el momento de la migración y durante los primeros años de residencia en España de acuerdo con la hipótesis de la interrupción. Posteriormente, se produce una recuperación de la fecundidad. El divergente comportamiento reproductivo de las distintas cohortes de edad a la llegada muestra la influencia del efecto interrupción y recuperación en el calendario reproductivo, especialmente en aquellas migrantes que llegaron en medio de su periodo fértil. La profundidad de estas tendencias depende de la región de origen y la edad a la llegada. Esta paralización del proceso reproductivo y de la formación familiar dentro del ciclo de vida debido al movimiento migratorio concuerda con resultados previos tanto para el conjunto de las inmigrantes en España (Devolder y Bueno, 2011; del Rey y Grande, 2017) como, por ejemplo, para el de las mujeres inmigrantes en Suecia (Anderson, 2004) o las inmigrantes hispanas en Estados Unidos (Parrado, 2011).

En tercer lugar, se observan diferentes comportamientos reproductivos de las migrantes en la sociedad de destino que vienen determinados por las diferentes características sociodemográficas y socioeconómicas, y particularmente las disímiles condiciones familiares. Uno de los principales hallazgos de este trabajo es la relevancia de la situación familiar como determinante del comportamiento reproductivo de las migrantes. Los resultados muestran que la fecundidad en España se encuentra favorecida por haber migrado sin hijos previos y por la convivencia con el cónyuge en destino.

Retomando la pregunta de investigación inicial y las hipótesis planteadas, los resultados muestran un cierto mantenimiento del patrón cultural, 
una fuerte interrupción de la fecundidad durante el proceso migratorio y un proceso de adaptación al comportamiento reproductivo de las mujeres españolas mediatizado por las condiciones socioeconómicas y familiares.

Finalmente, el actual contexto de crisis económica, con el freno de nuevas llegadas, la disminución del stock de inmigrantes latinoamericanos y los efectos del desempleo, deja abiertas futuras líneas de trabajo en relación a las pautas de fecundidad de la población migrante. Por ejemplo, resultaría interesante prestar atención al proceso de consolidación y asentamiento de la migración en España y su repercusión en las pautas de fecundidad y junto a ellos, analizar la posible convergencia o no de los modelos reproductivos en las segundas generaciones. En este sentido, el nuevo contexto económico que ha modificado la estructura de oportunidades también en lo relativo al comportamiento reproductivo, junto con el fin del "efecto llegada", explicaría el descenso de la fecundidad que se viene observando durante los últimos años tras el estallido de la crisis entre la población inmigrante.

\section{REFERENCIAS BIBLIOGRÁFICAS}

Abbasi-Shavazi, Mohammand J. y Mcdonald, Peter, 2002, “A comparison of fertility patterns of European immigrants in Australia with those in the countries of origin", in Genus, 58(1): 53-76.

Andersson, Gunnar, 2004, "Childbearing after migration: fertility patterns of foreign-born women in Sweden", in International Migration Review, 38(2): 747-774.

Bledsoe, C. H., Houle, René y Sow, Papa, 2007, "High fertility gambians in low fertility Spain: the dynamics of child accumulation cross transnational space", in Demographic Research, 16(12): 375-412.

Bongaarts, John, 2003, "Completing the fertility transition in the developing world: the role of educational differences and fertility preferences", in Population Studies, 57(3): 321-335.

Carter, Marion, 2000, "Fertility of Mexican immigrant women in the U.S.: a closer look", in Social Science Quarterly, 81(4): 1073-1086.

Castro-Martín, Teresa y Rosero-Bixby, Luis, 2011, "Maternidades y fronteras. La fecundidad de las mujeres inmigrantes en España", en RIS: Revista Internacional de Sociología, 69(1): 105-137.

Celade, 2001, “América Latina: fecundidad 1950-2050”, en Boletín Demográfico, núm. 68. División de Población de la CEPAL: Santiago de Chile.

Celade, 2013,: Proyecciones de Población. Observatorio Demográfico 2012, División de Población de la CEPAL. Santiago de Chile. 
Chackiel, Juan, 2004, "La dinámica demográfica en América Latina", en Serie Población y Desarrollo, núm. 52. CEPAL: Santiago de Chile.

Coleman, David A., 1994, "Trends in fertility and intermarriage among immigrant populations in western europe as measures of integration", in Journal of Biosocial Science, 26(1): 107-136.

Del Rey, Alberto y Cebrián, Mar, 2010, "Population Replacement and Migration inside Spain during the XXth century", in Population, 65(3): 481-497.

Del Rey, Alberto y Grande, Rafael, 2017, "El efecto de las condiciones de llegada en la reproducción de las mujeres migrantes", en Revista Española de Investigaciones Sociológicas, 159: 113-134.

Del Rey, Alberto y Grande, Rafael, 2015, “A longitudinal analysis of reproductive behaviour" in Domingo i Valls, A. Sabater, A. y Ruiz-Verdugo, R. (eds.) Demographic Analysis of Latin American Immigrants in Spain, Springer - Applied Demography Series vol. 5: 133-153.

Del Rey, Alberto; Cebrián, Mar, Grande, Rafael, Fernández-Macías, Enrique y Antón, José Ignacio, 2015, "La interferencia entre el estatus familiar y las características individuales en el nacimiento del primer hijo tras la emigración a España", Revista Internacional de Sociología , 73(2) pp. 1-13.

Devolder, Daniel y Bueno, Xiana, 2011, "Interacciones entre fecundidad y migración. Un estudio de las personas nacidas en el extranjero y residentes en Cataluña en 2007", en Documents d'Anàlisis Geogràfica, 57(3): 441-467.

Di Cesare, Mariachiara, 2007, “América latina: patrones emergentes en la fecundidad y la salud sexual y reproductiva y sus vínculos con la reducción de la pobreza", en Notas de Población, 84: 11-51.

Feliciano, Cynthia, 2005, "Educational selectivity in U.S. immigration: how do immigrants compare to those left behind?", in Demography, 42(1):131-152.

Ford, Kathleen, 1990, "Duration of Residence in the United States and the Fertility of U.S. Immigrants", in International Migration Review, 24(1): 34-68.

Goldstein, Sidney, 1973, "Interrelations between migration and fertility in Thailand", in Demography, 10(2): 225-241.

Grande, Rafael, 2012, España en el camino de Bolivia. Pautas de selectividad y procesos de inserción de la reciente migración boliviana a España. Berlín: Lap Lambert Academic Publishing.

Grande, Rafael y del Rey, Alberto, 2012, "Remesas, proyectos migratorios y relaciones familiares. El caso de los latinoamericanos y los caribeños en España", en Papeles de Población, 18(74): 237-272.

Kahn, Joan R., 1988, "Immigrant selectivity and fertility adaptation in the United States", in Social Forces, 67(1): 108-128.

Kahn, Joan R., 1994, "Immigrant and native fertility during the 1980s: adaptation and expectations for the future", in The International Migration Review, 28(3): 501-519. 
Kulu, Hill, 2005, "Migration and fertility: competing hypotheses re-examined", in European Journal of Population / Revue Européenne de Démographie, 21(1): 51-87.

Lindstrom, David P. y Giorguli, Silvia, 2007, "The interrelationship of fertility, family maintenance and Mexico-U.S. Migration”, in Demographic Research, 17: 28-28.

Milewski, Nadja, 2007, "First child of immigrant workers and their descendants in West Germany: interrelation of events, disruption, or adaptation?", in Demographic Research, 17(29): 859-896.

Muñoz de Bustillo, Rafael y Antón, José Ignacio, 2010, “De la España que emigra a la España que acoge: contexto, dimensión y características de la migración latinoamericana en España", en América Latina Hoy, 55: 15-39.

Mussino, Eleonora y Strozza, Salvatore, 2012, "The fertility of immigrants after arrival: the Italian case", in Demographic Research, 26(4): 99-130.

Parrado, Emilio, 2011, "How High is Hispanic/Mexican Fertility in the United States? Immigration and Tempo Considerations", in Demography, 48(3): 10591080 .

Parrado, Emilio y Morgan, Philip, 2008, “Intergenerational Fertility among Hispanic Women: New Evidence of Immigrant Assimilation”, in Demography, 45(3): 651-671.

Portes, Alejandro y Zhou, Min, 1993, “The new second generation: segmented assimilation and its variants", in Annals of the American Academy of Political and Social Sciences 530: 74-96.

Roig-Vila, Marta y Castro-Martín, Teresa, 2007, "Childbearing patterns of foreign women in a new immigration country: the case of Spain", in Population, 62(3): 351-379.

Rosero-Bixby, Luis, 2004, "La fecundidad en áreas metropolitanas de América Latina: la fecundidad de reemplazo y más allá”, en Notas de Población, 78: 35-63.

Sobotka, Tomás, 2008, "The rising importance of migrants for childbearing in Europe", in Demographic Research, 19(9): 225-248.

Stark, Oded, 1991, The Migration of Labor. Cambridge: Mass.

Stephen, Elizabeth H. y Bean, Frank D., 1992, “Assimilation, Disruption and the Fertility of Mexican-Origin Women in the United States", in International Migration Review, 26(1): 67-88.

Toulemon, Laurent, 2004, "Fertility among Immigrant Women: New Data, a New Approach", in Population \& Societies, 400: 1-4. 


\section{RESUMEN CURRICULAR DE LOS AUTORES}

\section{Rafael Grande}

Doctor en Ciencias Sociales y Máster en Estudios Latinoamericanos por la Universidad de Salamanca. Actualmente es profesor ayudante doctor en la Universidad de Málaga. Ha dirigido el proyecto "Asimilación laboral e integración social en flujos migratorios sur-sur y sur-norte. El caso de América Latina y el Caribe" financiado por Ministerio de Economía y Competitividad de España. Sus líneas de investigación se centran en el estudio de la integración laboral y familiar de la población inmigrantes y del fenómeno de las remesas. Previamente ha realizado estancias de investigación en el Centre d'Estudis Demogràfics (Barcelona, España), en la Office of Population Research de Princeton University (New Jersey, Estados Unidos) y en la Universidad Autónoma de Chiapas (México).

Dirección electrónica: rgrande@uma.es

\section{Alberto del Rey}

Doctor en Demografía por la Universidad Autónoma de Barcelona. Actualmente Profesor Titular en el departamento de Sociología de la Universidad de Salamanca y miembro del Instituto de Iberoamérica de la Universidad de Salamanca. Sus líneas de investigación se centran en el estudio del comportamiento reproductivo y la integración de la población inmigrante, temas sobre los que he dirigido varios proyectos de investigación y he realizado distintas publicaciones en revistas internacionales. Anteriormente ha realizado estancias de investigación en el CIESAS (México), en la Universidad Autónoma de Barcelona, en el Centro de Estudios Demográficos, en el Max Planck Institute for Demographic Research (Alemania), en el Population Studies Center de la Universidad de Pennsylvania (Philadelphia, EEUU) y en Institute de Recherche pour le Developpement (Paris, Francia).

Dirección electrónica: adelrey@usal.es

Artículo recibido el 8 de enero de 2016 y aprobado el 23 de marzo de 2017. 